\title{
Propriedades psicométricas da Escala de Respostas Socialmente Desejáveis - 5 (SDRS-5) em jovens institucionalizados
}

\author{
Pedro Pechorro1, Ricardo Barroso 2,3, Irene Silva4, João Marôco4, Rui Abrunhosa \\ Gonçalves ${ }^{1}$ \\ ${ }^{1}$ Escola de Psicologia, Universidade do Minho \\ 2 Universidade de Trás-os-Montes e Alto Douro \\ 3 Universidade do Porto \\ ${ }^{4}$ ISPA - Instituto Universitário
}

\begin{abstract}
Resumo: 0 objetivo do presente estudo consistiu em analisar as propriedades psicométricas da Escala de Respostas Socialmente Desejáveis - 5 (SDRS-5) em uma amostra de jovens institucionalizados. Com base nesta amostra $(N=221)$, a versão portuguesa da SDRS-5 demonstrou boas propriedades psicométricas, nomeadamente em termos de estrutura fatorial, consistência interna, validade convergente e validade discriminante que de uma forma geral justificam a sua utilização. Em termos de validade de critério, foram encontradas correlações negativas entre a SDRS-5 e consumo de álcool, de cannabis, de cocaína/heroína e sexo sem preservativo.
\end{abstract}

Palavras-chave: Avaliação; Adolescência; Desejabilidade social; Validação.

Psychometric properties of the Socially Desirable Response Set Measure (SDRS-5) among institutionalized youths: The main aim of the present study was to examine the psychometric properties of the Socially Desirable Response Set Measure (SDRS-5) among a sample of institutionalized youths. Based on this sample $(N=221)$, the Portuguese version of the SDRS-5 demonstrated good psychometric properties, namely in terms of factor structure, internal consistency, convergent validity, and discriminant validity that support its use for Portuguese adolescents samples. In terms of criterionrelated validity, negative correlations were found between SDRS-5 scores and alcohol use, cannabis use, cocaine/heroin use, and unprotected sex.

Keywords: Assessment; Adolescence; Social desirability; Validation.

Os investigadores que utilizam questionários e entrevistas baseiam-se nas respostas dos participantes para elaborar as suas inferências sobre os constructos latentes que estes questionários operacionalizam. Todavia, alguns participantes apresentam uma tendência ou viés de resposta designada por desejabilidade social, que leva a que, conscientemente ou não, apresentem uma imagem deles próprios distorcida de forma favoravelmente positiva. Os participantes podem acreditar que a informação que estão a fornecer é verdadeira (self-deception) ou então estarem a falsificá-la propositadamente (impression management) para simularem em conformidade com os valores socialmente aceitáveis, ganhando aprovação social ou evitando ser alvo de críticas (King \& Brunner 2000; Paulhus, 1984; van de Mortel, 2008). Alguns autores (e.g., Nederhof, 1985) estimam que a desejabilidade social pode explicar entre $10 \%$ a $75 \%$ da variância das respostas dos participantes, contribuindo decisivamente para obscurecer as relações entre as variáveis sob estudo.

Tem havido uma longa discussão na literatura sobre se a desejabilidade social é um traço de personalidade ou uma estratégia situacional, sendo que a investigação mais recente indica que a maioria da variância das respostas é atribuível à diferença no traço e apenas uma pequena, mas significativa proporção da variância é devida às condições situacionais (Crutzen \& Goritz, 2010). Todavia, ainda subsistem questões relacionadas com a desejabilidade social que não foram devidamente esclarecidas. De acordo com Holtgraves (2004), o facto de diferentes medidas de desejabilidade social não se correlacionarem fortemente umas com as outras indicia que estão subjacentes diferentes conceptualizações deste constructo. Também não se conhece bem a forma como a desejabilidade social funciona: será um processo consciente e deliberado ou, pelo contrário, inconsciente e automático? Independentemente destas questões, desde um ponto de vista prático, se as medidas de autorresposta

${ }^{1}$ Dados de contacto para correspondência: Pedro Fernandes dos Santos Pechorro. Escola de Psicologia, Universidade do Minho, Campus de Gualtar, 4710-057 Braga. Portugal. E-mail: ppechorro@gmail.com. 
são de facto influenciadas pela desejabilidade social, então o seu controlo pode remover algum do erro e melhorar a validade deste tipo de medidas.

Um dos instrumentos mais utilizados para medir o constructo da desejabilidade social é a Escala de Desejabilidade Social de Marlowe-Crowne (Marlowe-Crowne Social Desirability Scale - MCSDS; Crowne \& Marlowe, 1960; Johnston, Wright, \& Weinman, 1995). Esta escala clássica foi originalmente construída com 33 itens em formato de resposta dicotómico (Verdadeiro/Falso), selecionados por se considerar que representariam comportamentos culturalmente aprovados com uma baixa probabilidade de ocorrência e terem implicações psicopatológicas/anormais mínimas. A MCSDS tem como objetivo avaliar a tendência que certas pessoas têm em apresentar as suas qualidades de forma inflacionada ou exagerada, minimizando simultaneamente as suas fraquezas (i.e., tentarem apresentar-se a si próprias como estando dentro dos ideais das normas da sociedade em que se inserem). Devido ao elevado número de itens da MCSDS original, não tardou muito para que outros investigadores explorassem a estrutural fatorial da MCSDS e surgissem versões curtas de aplicação menos demorada. Seguidamente iremos rever algumas delas.

Strahan e Gerbasi (1972), utilizando uma amostra constituída por 361 estudantes, efetuaram uma análise de componentes principais aos 33 itens da MCSDS que levou à obtenção de dois fatores, cada um com dez itens, designados X1 e X2. A consistência interna destes dois fatores variou entre 0,28 e 0,54 nos vários estudos de validação que os autores efetuaram, valores considerados excessivamente baixos. Reynolds (1982), recorrendo a uma amostra de 608 estudantes universitários, efetuou também análise de componentes principais da MCSDS e correlações dos itens com o total da escala. 0 autor identificou três fatores que designou por $\mathrm{A}, \mathrm{B}$ e $\mathrm{C}$, cada um deles com respetivamente 11 itens, 12 itens e 13 itens, sendo que a consistência interna destes fatores variou entre 0,74 e 0,76. Ballard (1992), recorrendo a uma amostra de 399 estudantes universitários, construiu várias versões curtas da MCSDS, sendo que a mais difundida em termos de utilização ficou conhecida como subescala compósita ou MCSDS-SF. Esta autora, utilizando análise de componentes principais, selecionou os 13 itens da MCSDS-SF, que demonstrou ser unidimensional e ter uma consistência interna de 0,70.

Hays, Hayashi e Stewart (1989) propuseram-se construir uma versão muito curta de desejabilidade social derivada da MCSDS que demorasse menos de um minuto a preencher de forma a poder ser utilizada rotineiramente em avaliações sem grande consumo de tempo. Para tal, pegaram na forma A desenvolvida por Reynolds (1982), com uma estrutura fatorial unidimensional, com o objetivo de reduzir o número de itens. 0 formato dicotómico dos itens foi alterado para ordinal de cinco pontos de forma a facilitar a resposta e melhorar a sensibilidade da medida. Foi utilizada uma amostra de 614 pacientes externos, seguida de uma amostra de validação cruzada de 3053 pacientes externos. Apenas os 5 itens com as maiores correlações item-total foram incluídos na nova medida, que Hays et al. (1989) designaram por Escala de Respostas Socialmente Desejáveis - 5 (SDRS-5). A nova medida demonstrou correlações item-total entre 0,35 e 0,49, alfas de Cronbach entre 0,66 e 0,68 e fiabilidade teste-reteste a um mês de 0,75 , todavia os autores não forneceram quaisquer dados sobre a estrutura fatorial nem demais propriedades psicométricas.

As investigações psicométricas que se debruçaram sobre as várias formas curtas da MCSDS não permitem tirar conclusões consistentes dado que algumas reportam bons resultados (e.g., Andrews \& Meyer, 2003; Loo \& Thorpe, 2000) enquanto outras reportam resultados claramente aquém do satisfatório (e.g., Barger, 2002). No que diz respeito às adaptações transculturais, um estudo recente de Verardi et al. (2010) sugere que a forma C da versão curta da MCSDS não deve ser utilizada por não demonstrar equivalência, mas deixa em aberto a possibilidade de se criar uma escala que seja aplicável em diferentes países.

Em Portugal existe uma enorme necessidade de proceder à validação de instrumentos psicométricos aplicáveis à área da adolescência em geral e da delinquência juvenil em particular de forma a fundamentar empiricamente a sua utilização na realidade nacional. 0 objetivo do presente artigo, englobado no âmbito de uma investigação mais abrangente sobre delinquência juvenil, consistiu em adaptar e estudar as qualidades psicométricas da SDRS-5 numa amostra de adolescentes institucionalizados por ordem do tribunal devido a comportamentos delituosos. Neste processo foram colocadas algumas hipóteses: a) espera-se confirmar a estrutura unidimensional da SDRS-5 através de análise fatorial confirmatória; b) espera-se que a SDRS-5 demonstre uma consistência interna satisfatória; c) espera-se que a SDRS-5 demonstre validade convergente com outra medida de desejabilidade social, bem como validade discriminante com medidas de empatia e ansiedade social; d) espera-se que a SDRS-5 demonstre validade de critério com variáveis como o consumo de álcool, de drogas e sexo desprotegido (i.e., sem preservativo). 


\section{MÉTODO}

\section{Participantes}

A amostra foi recolhida nos Centros Educativos existentes a nível nacional em Portugal, que são geridos pela Direção-Geral de Reinserção e Serviços Prisionais (DGRSP). Um total de 221 participantes do sexo masculino $(M=16.75$ anos; $D P=1.41$ anos; amplitude $=13-20$ anos $)$ concordou em participar voluntariamente na presente investigação. Os participantes tinham uma proveniência predominantemente urbana (92.8\%), eram predominantemente europeus (54.3\%) em termos étnicos. Os jovens encontravam-se internados por ordem judicial, sendo que o internamento é a medida tutelareducativa mais gravosa que o tribunal pode decretar.

0 início na atividade criminal ocorreu precocemente nas vidas destes jovens $(M=11.33$ anos, $D P=$ 2.24 anos), tendo sido detidos na sua maioria antes de terem 16 anos $(M=15.46$ anos, $D P=1.31$ anos $)$ e sido condenados a uma média de 21 meses de internamento $(M=20.67$ meses, $D P=6.69$ meses $)$. A maioria destes jovens (87.6 \%) foi internada devido a terem cometido crimes graves/violentos (e.g., roubo, agressão, violação).

\section{Instrumentos}

A Escala de Respostas Socialmente Desejáveis - 5 (Socially Desirable Response Set 5 - SDRS-5; Hays et al., 1989) é uma medida breve de autorresposta que avalia o constructo de desejabilidade social. A SDRS- 5 pode ser cotada somando os 5 itens ordinais de 5 pontos de Totalmente verdadeiro (1) a Totalmente falso (5), após se ter efetuado a reversão dos itens 2, 3 e 4. Hays et al. (1989) referem que os itens podem ser misturados aleatoriamente com os itens de outras escalas mesmo que tenham formatos de resposta diferentes (e.g., itens ordinais de 4 pontos), e ser posteriormente dicotomizados (Totalmente verdadeiro $=$ 1 , restantes opções de resposta $=0$ ) de forma a maximizar a validade da escala. Pontuações mais altas indicam níveis de desejabilidade social mais altos.

A Escala de Desejabilidade Social de Marlowe-Crowne (Marlowe-Crowne Social Desirability Scale Versão Curta - MCSDS-SF; Ballard, 1992) foi concebida a partir da escala original de Marlowe-Crowne, (Crowne \& Marlowe, 1960), tendo ficado conhecida como subescala compósita e sendo provavelmente a mais conhecida de todas as versões curtas existentes. A MCSDS-SF pode ser cotada simplesmente somando os itens dicotómicos (Verdadeiro/Falso) que a compõem após se ter efetuado a reversão dos itens apropriados. Pontuações mais elevadas refletem a tendência de dar respostas socialmente mais desejáveis. Na presente investigação foi utilizada a versão portuguesa da MCSDS-SF (Pechorro, Vieira, Poiares, \& Marôco, 2012). A consistência interna por coeficiente de Kuder-Richardson da MCSDS-SF para a presente investigação foi de .61 .

A Escala de Empatia Básica (Basic Empathy Scale - BES; Jolliffe \& Farrington, 2006) é uma medida de autorresposta constituída por 20 itens, desenhada para medir duas dimensões da empatia em jovens: empatia afetiva e empatia cognitiva. Cada item é cotado em escala ordinal de 5 pontos, de Discordo totalmente a Concordo totalmente. A pontuação total e as pontuações de cada dimensão são obtidas somando os respetivos itens. Pontuações mais elevadas indicam níveis de empatia mais elevados. $\mathrm{Na}$ presente investigação foi utilizada a versão portuguesa da BES (Pechorro, Ray, Salas-Wright, Marôco, \& Gonçalves, 2015). A consistência interna, estimada por alfa de Cronbach, da BES para a presente investigação foi de 91 .

A Escala de Ansiedade Social para Adolescentes (Social Anxiety Scale for Adolescents - SAS-A; La Greca, 1998; La Greca \& Lopez, 1998) é uma medida de autorresposta constituída por 22 itens (dos quais 4 são itens neutros não cotados) que avalia as experiências de ansiedade social dos adolescentes no contexto das relações com os seus pares. Os itens são avaliados segundo uma escala ordinal de 5 pontos, que vai de De forma nenhuma a Todas as vezes. Para além da pontuação total podem-se obter também as pontuações nas três subescalas, nomeadamente: FNE, SAD-Novo e SAD-Geral (La Greca \& Lopez, 1998). Pontuações mais elevadas indicam níveis de ansiedade social mais elevados. Na presente investigação foi utilizada a versão portuguesa da SAS-A (Pechorro, Silva, Marôco, \& Gonçalves, 2014). A consistência interna, estimada por alfa de Cronbach, da SAS-A para a presente investigação foi de .91.

Adicionalmente foi construído um questionário para descrever as variáveis sociodemográficas dos participantes. Este questionário incluiu variáveis como idade, nacionalidade, grupo étnico, proveniência rural versus urbana, nível de escolaridade concluído, estado civil dos pais, consumo de álcool, consumo de cannabis, consumo de cocaína/heroína e sexo desprotegido (i.e., sexo sem preservativo).

\section{Procedimentos}

Foi solicitada permissão da RAND Corporation para validar uma versão portuguesa da SDRS-5, tendo esta organização informado que a escala é de utilização livre e disponibilizada publicamente sem custos. No processo de tradução e de retroversão foram seguidos os procedimentos adequados (Hambleton, 
Merenda, \& Spielberger, 2005). A tradução inicial de inglês para português foi efetuada pelos autores deste artigo, seguida da retroversão por um tradutor especialista. Não foram encontradas diferenças relevantes entre a retroversão e a versão original, ficando demonstrado que os itens traduzidos tinham um significado idêntico ou muito semelhante aos itens originais em inglês.

A Direcção-Geral de Reinserção e Serviços Prisionais (DGRSP) concedeu autorização para avaliar os jovens internados nos Centros Educativos existentes a nível nacional. Os potenciais participantes foram informados sobre os objetivos do estudo, sendo salientado que a participação era voluntária, anónima e confidencial. Nem todos os jovens abordados participaram no estudo, nomeadamente devido a: recusa em ser avaliado, não ser fluente na língua portuguesa e isolamento por questões de segurança. A avaliação foi feita através de entrevistas individuais em contexto apropriado. Foram também utilizados dados constantes no processo de internamento dos jovens.

Os dados foram analisados utilizando o SPSS v22 (IBM SPSS, 2013) e o EQS 6.2 (Bentler \& Wu, 2008). A estrutura fatorial da versão Portuguesa da SDRS-5 foi avaliada com Análise Fatorial Confirmatória (AFC) efetuada no software EQS com métodos robustos de estimação. Os índices de ajustamento calculados incluíram: Qui-quadrado de Satorra-Bentler/graus de liberdade, CFI (Comparative Ft Index), IFI (Incremental Fit Index), RMSEA (Root Mean Square Error of Approximation). Um qui-quadrado/graus de liberdade $<5$ é considerado adequado, $\leq 2$ é considerado bom e valores $=1$ são considerados muito bons (Marôco, 2014; West, Taylor, \& Wu, 2012). Considerou-se que CFI $\geq 0.90$ e RMSEA $\leq 0.08$ indicam um ajustamento adequado, enquanto CFI $\geq 0.95$ e RMSEA $\leq 0.06$ indicam um ajustamento bom (Byrne, 2006). Um IFI $\geq 0.90$ é considerado aceitável. Em caso de necessidade, seriam utilizados Índices de Modificação para melhorar o ajustamento (Marôco, 2014).

A AFC foi efetuada diretamente nos itens da SDRS-5 e somente valores com saturação $\geq 0.45$ foram considerados. Optou-se pela utilização de correlações policóricas nos itens ordinais porque estas proporcionam melhores resultados (Holgado-Tello, Chacón-Moscoso, Barbero-García, \& Vila-Abad, 2010). Correlações Pearson foram utilizadas para analisar as associações entre variáveis escalares, enquanto as correlações bisseriais por ponto foram utilizadas para analisar a relação entre variáveis nominais dicotómicas e variáveis escalares (Leech, Barrett, \& Morgan, 2015; Tabachnick \& Fidell, 2007).

\section{RESULTADOS}

0 primeiro passo na análise das propriedades psicométricas da versão portuguesa da SDRS-5 foi a tentativa de replicação da estrutura fatorial através de análise fatorial confirmatória. Utilizando o método ML Robust obtivemos resultados em termos de índices de ajustamento que confirmam a presença da estrutura fatorial unidimensional postulada originalmente por Hays et al. (1989), nomeadamente: S$\mathrm{B} \chi^{2} / \mathrm{df}=12.66 / 5 ; \mathrm{IFI}=0.98 ; \mathrm{CFI}=0.97 ;$ RMSEA $(90 \% \mathrm{CI})=0.08(0.03-0.14)$. Na Tabela 1 apresentamos os pesos fatoriais obtidos na nossa amostra, sendo de salientar que todos os itens tiveram pesos acima de 0.45 .

Tabela 1. Saturações dos itens da SDRS-5 por análise fatorial confirmatória

\begin{tabular}{ll}
\hline Itens SDRS-5 & Fator $\mathbf{1}$ \\
\hline 1. Sou sempre simpático, mesmo com pessoas que são mal-educadas. & 0.48 \\
2. Já me aconteceu aproveitar-me de outras pessoas para meu ganho pessoal. (R) & 0.72 \\
3. Por vezes tento vingar-me em vez de perdoar e esquecer. (R) & 0.64 \\
4. Por vezes fico chateado quando não consigo o que quero. (R) & 0.65 \\
5. Ouço sempre com muita atenção todas as pessoas com quem falo. & 0.55 \\
\hline
\end{tabular}

Nota. SDRS-5 = Escala de Respostas Socialmente Desejáveis - 5; (R) = item reversível

O passo seguinte consistiu na estimação do Alfa de Cronbach que para itens dicotómicos é igual ao Kuder-Richardson (Marôco \& Garcia-Marques, 2006) das correlações inter-itens e da amplitude das correlações item-total corrigidas da SDRS-5 (ver Tabela 2).

Tabela 2. Alfa de Cronbach, média das correlações inter-itens e amplitude das correlações item-total corrigidas para a SDRS-5

\begin{tabular}{lccc}
\hline & $\alpha$ & MCII & ACITC \\
\hline SDRS-5 & .73 & 0.35 & $0.37-0.8$ \\
\hline Nota. SDRS-5 = Escala de Respostas Socialmente Desejáveis $-5 ; \alpha$ Cronbach = Alfa de Cronbach; MCII = Médias das correlações
\end{tabular}

inter-itens; ACITC = Amplitudes das correlações item-total corrigidas

A validade convergente da SDRS-5 foi efetuada com a MCSDS-SF, enquanto a validade discriminante foi efetuada com a BES e a SAS-A (ver Tabela 3). 
Tabela 3. Validade convergente da SDRS-5 com a MCSDS-SF e validade discriminante com a BES e a SAS-A

\begin{tabular}{|c|c|c|c|}
\hline & MCSDS-SF & BES & SAS-A \\
\hline SDRS-5 & $0.81^{* * *}$ & $-0.09^{n s}$ & $-0.10^{n s}$ \\
\hline
\end{tabular}

Foram também efetuadas correlações com variáveis associadas a comportamentos de risco em jovens, tais como consumo de álcool, drogas e sexo desprotegido (i.e., sem preservativo), como pode ser constatado na Tabela 4.

Tabela 4. Correlações da SDRS- 5 com as variáveis consumo de álcool, consumo de cannabis, consumo de cocaína/heroína e sexo sem preservativo

\begin{tabular}{lcccc}
\hline & Álcool & Cannabis & Cocaína/heroína & Sexo sem preservativo \\
\hline SDRS-5 & $-0.30^{* * *}$ & $-0.32^{* * *}$ & $-0.26^{* * *}$ & $-0.16^{*}$ \\
\hline $\begin{array}{l}\text { Nota. SDRS-5 = Escala de Respostas Socialmente Desejáveis }-5 \\
* * * \text { significativo ao nível } 0.001 ; * \text { significativo ao nível } 0.05\end{array}$ & &
\end{tabular}

\section{DISCUSSÃO}

O presente estudo teve por objetivo principal analisar as propriedades psicométricas da SDRS-5 numa amostra de adolescentes institucionalizados. A utilização de análise fatorial confirmatória permitiu confirmar a estrutura unidimensional postulada pelos autores da versão americana original (Hays et al., 1998) na nossa amostra portuguesa. 0 ajustamento do modelo unidimensional foi considerado bom à luz dos índices de ajustamento, incluindo os pesos fatoriais dos itens nas respetivas dimensões que estiveram sempre acima de 0,45. Desta forma, consideramos que se confirmou a nossa primeira hipótese.

A análise da consistência interna através de alfa de Cronbach revelou um valor de .73 claramente acima do limite mínimo recomendado de 70 (Cortina, 1993; Kaplan \& Saccuzzo, 2013) para estudos exploratórios (Marôco \& Garcia-Marques, 2006), tendo o valor sido inclusivamente melhor que o obtido originalmente por Hays et al. (1989). Em termos da média das correlações inter-itens, a SDRS-5 total obteve um valor dentro do intervalo recomendado de 0.15 a 0.50 , o que indicia uma adequada homogeneidade dos itens que a constituem (Clark \& Watson, 1995). Em termos das correlações item-total corrigidas, a SDRS-5 obteve valores sempre acima do valor recomendado de 0.30 (Kaplan \& Saccuzzo, 2013; Nunnally \& Bernstein, 1994), sendo que estes podem ser considerados inclusivamente melhores que os obtidos por Hays et al. (1989) por serem mais elevados. Tais resultados levam-nos a considerar que a segunda hipótese colocada foi confirmada.

A validade convergente da SDRS-5 com a MCSDS-SF revelou uma correlação alta positiva e estatisticamente significativa, antecipada também pelo facto de ambas as medidas serem derivadas da MCSDS original; ficou assim demonstrada a esperada sobreposição entre estas duas medidas de desejabilidade social (AERA, APA, \& NCME, 2014). A validade discriminante da SDRS-5 com a BES (Jolliffe \& Farrington, 2004; Kämpfe, Penzhorn, Schikora, Dünzl, \& Schneidenbach, 2009) e a SAS-A (Dadds, Perrin, \& Yule, 1998; Egloff \& Schmukle, 2003) revelou as esperadas correlações baixas ou nãosignificativas (Kaplan \& Saccuzzo, 2013). A terceira hipótese foi, portanto, também confirmada.

As correlações da SDRS-5 com consumo de álcool, consumo de cannabis, consumo de cocaína/heroína e sexo desprotegido (i.e., sem preservativo) foram negativas e estatisticamente significativas, revelando a esperada tendência em minimizar tais comportamentos de risco socialmente indesejáveis intrinsecamente associada à desejabilidade social (Mensch \& Kendel, 1988; Welte \& Russell, 1993). Tal leva-nos a concluir que também a quarta hipótese foi confirmada.

As questões de self-deception e de impression management relacionadas com o constructo de desejabilidade social são muito relevantes no processo de avaliação psicológica, particularmente quando se trata de avaliação psicológica em contexto forense, em que é sabido que os avaliados têm uma forte tendência a distorcer as suas respostas. 0 controlo da desejabilidade social pode remover algum do erro e melhorar a validade de medidas de autorresposta. Os resultados do nosso estudo levam-nos a concluir que a SDRS-5 é um instrumento válido e fiável na medição da desejabilidade social, podendo ser utilizado no processo de avaliação de jovens portugueses.

Todavia, devemos mencionar algumas limitações do nosso estudo. 0 facto de se ter utilizado a MCSDS-SF para efetuar a validade convergente limitou o alcance das conclusões dado que esta também é derivada da MCSDS original. 0 facto de termos utilizado apenas jovens do sexo masculino detidos em centro educativo também foi uma limitação por faltar diversidade a esta amostra. No futuro será necessário analisar as propriedades psicométricas da SDRS-5 em outras amostras (e.g., amostras 
escolares, amostras clínicas, amostras com participantes do sexo masculino e do sexo feminino) de forma a demostrar que os resultados agora obtidos são generalizáveis. Será necessário também efetuar outros tipos de procedimentos psicométricos, como a avaliação da estabilidade temporal (teste-reteste) e a validade convergente com outras medidas específicas de desejabilidade social, especialmente as que não sejam derivadas da MCSDS original.

\section{Referências}

American Educational Research Association (AERA), American Psychological Association (APA), \& National Council for Measurement in Education (NCME) (2014). Standards for Educational and Psychological Testing. Washington, DC: AERA.

Andrews, P., \& Meyers, R. (2003). Marlowe-Crowne Social Desirability Scale and Short Form C: Forensic Norms. Journal of Clinical Psychology, 59(4), 483-492. http://dx.doi.org/10.1002/jclp.10136

Ballard, R. (1992). Short forms of the Marlowe-Crowne Social Desirability Scale. Psychological Reports, 71, 1155-1160. doi: 10.2466/PR0.71.8.1155-1160

Barger, S. (2002). The Marlowe-Crowne affair: Short forms, psychometric structure and social desirability. Journal of Personality Assessment, 79(2), 286-305. doi: 10.1207/S15327752JPA7902_11

Bentler, P., \& Wu, E. (2008). EQS for Windows user's guide. Encino, CA: Multivariate Software, Inc.

Byrne, B. (2006). Structural equation modeling with EQS: Basic concepts, applications, and programming. Mahwah, NJ: Lawrence Erlbaum Associates.

Clark, L., \& Watson, D. (1995). Constructing validity: Basic issues in objective scale development. Psychological Assessment, 7(3), 309-319. http://dx.doi.org/10.1037//1040-3590.7.3.309

Cortina, J. (1993). What is coefficient alpha? An examination of theory and applications. Journal of Applied Psychology, 78(1), 98-104. doi: 10.1037//0021-9010.78.1.98

Crowne, D., \& Marlowe, D. (1960). A new scale of social desirability independent of psychopathology. Journal of Consulting Psychology, 24, 349-354. http://dx.doi.org/10.1037/h0047358

Crutzen, R., \& Goritz, A. (2010). Social desirability and self-reported health risk behaviors in web-based research: Three longitudinal studies. BMC Public Health, 10, 720. doi: 10.1186/1471-2458-10-720

Dadds, M. R., Perrin, S., \& Yule, W. H. (1998). Social desirability and self-reported anxiety in children: an analysis of the RCMAS Lie scale. Journal of Abnormal Child Psychology, 26, 311-317. doi: 10.1023/A:1022610702439

Egloff, B., \& Schmukle, S. C. (2003). Does social desirability moderate the relationship between implicit and explicit anxiety measures? Personality and Individual Differences, 35, 1697-1706. doi: 10.1016/S0191-8869(02)00391-4

Hambleton, R., Merenda, P., \& Spielberger, C. (2005). Adapting educational and psychological tests for cross-cultural assessment. Mahwah, NJ: Lawrence Erlbaum Associates.

Hays, R., Hayashi, T., \& Stewart, A. (1989). A five-item measure of Socially Desirable Response Set. Educational and Psychological Measurement, 49(3), 629-636. doi: 10.1177/001316448904900315

Holgado-Tello, F., Chacón-Moscoso, S., Barbero-García, I., \& Vila-Abad, E. (2010). Polychoric versus Pearson correlations in exploratory and confirmatory factor analysis of ordinal variables. Quality \& Quantity, 44(1), 153-166. doi: 10.1007/s11135-008-9190-y

Holtgraves, T. (2004). Social desirability and self-reports: Testing models of socially desirable responding. Personality and Social Psychology Bulletin, 30(2), 161-172. doi: 10.1177/0146167203259930

Jolliffe, D., \& Farrington, D. (2006). Development and validation of the Basic Empathy Scale. Journal of Adolescence, 29(4), 589-611. doi: 10.1016/j.adolescence.2005.08.010

Johnston, M., Wright, S., \& Weinman, J. (1995). Measures in health psychology: A user's portfolio. Windsor (UK): NFER-NELSON Publishing Company Ltd.

Kämpfe, N., Penzhorn, J., Schikora, J., Dünzl, J., \& Schneidenbach, J. (2009). Empathy and social desirability: A comparison of delinquent and non-delinquent participants using direct and indirect measures. Psychology, Crime \& Law, 15(1), 1-17. doi: 10.1080/10683160802010640

Kaplan, R., \& Saccuzzo, D. (2013). Psychological testing: Principles, applications, and issues (8 $8^{\text {th }}$ ed.). Belmont, CA: Wadsworth, Cengage Learning.

King, M., \& Bruner, G. (2000). Social desirability bias: A neglected aspect of validity testing. Psychology and Marketing, 17(2), 79-103. doi: 10.1002/(SICI)1520-6793(200002)17:2<79::AID-MAR2>3.0.CO;2-0

La Greca, A. (1998). Manual for the Social Anxiety Scales for Children and Adolescents. Miami, FL: University of Miami.

La Greca, A., \& Lopez, N. (1998). Social anxiety among adolescents: Linkages with peer relations and friendships. Journal of Clinical Child Psychology, 26, 83-94. doi: 10.1023/A:1022684520514 
Leech, N., Barrett. K., \& Morgan, G. (2015). IBM SPSS for intermediate statistics (5 ${ }^{\text {th }}$ ed.). New York, NY: LEA.

Loo, R., \& Thorpe, K. (2000). Confirmatory factor analyses of the full and short versions of the MarloweCrowne Social Desirability Scale. The Journal of Social Psychology, 140(5), 628-635. doi: $10.1080 / 00224540009600503$

Marôco, J. (2014). Análise de Equações Estruturais: Fundamentos teóricos, software \& aplicações (2a ed.). Pero Pinheiro: ReportNumber, Lda.

Marôco, J., \& Garcia-Marques, T. (2006). Qual a fiabilidade do alfa de Cronbach? Questões antigas e soluções modernas? Laboratório de Psicologia, 4, 65-90.

Mensch, B. S., \& Kendel, D. B. (1988). Underreporting of substance use in a national longitudinal youth cohort: Individual and interviewer effects. Public Opinion Quarterly, 52, 100-124. doi: $10.1086 / 269084$

Nederhof, A. (1985). Methods of coping with social desirability bias: A review. European Journal of Social Psychology, 15(3), 263-280. doi: 10.1002/ejsp.2420150303

Nunnally, J., \& Bernstein, I. (1994). Psychometric theory (3rd ed.). New York: McGraw-Hill.

Paulhus, D. (1984). Two component models of socially desirable responding. Journal of Personality and Social Psychology, 46(3), 598-609. doi: 10.1037/0022-3514.46.3.598

Pechorro, P., Vieira, R., Poiares, C., \& Marôco, J. (2012). Contributos para a validação duma versão curta da Escala de Desejabilidade Social de Marlowe-Crowne com adolescentes portugueses. Arquivos de Medicina, 26(1), 11-17.

Pechorro, P., Silva, I., Marôco, J., \& Gonçalves, R. (2014). Propriedades psicométricas da Escala de Ansiedade Social para Adolescentes em jovens institucionalizados Psicologia, Saúde \& Doenças, 15(3), 586-596. doi: 10.15309/14psd150303

Pechorro, P., Ray, J., Salas-Wright, C., Marôco, J., \& Gonçalves, R. (2015). Adaptation of the Basic Empathy Scale among a Portuguese sample of incarcerated juvenile offenders. Psychology, Crime \& Law, 21(7), 699-714. doi: 10.1080/1068316X.2015.1028546

Reynolds, W. (1982). Development of reliable and valid short forms of the Marlowe-Crowne Social Desirability Scale. Journal of Clinical Psychology, 38, 119-125. doi: 10.1002/10974679(198201)38:1\%3C119::AID-JCLP2270380118\%3E3.0.C0;2-I

Strahan, R., \& Gerbasi, K. (1972). Short, homogeneous versions of the Marlowe-Crowne Social Desirability Scale. Journal of Clinical Psychology, 28, 191-193. doi: 10.1002/10974679(197204)28:2\%3C191::AID-JCLP2270280220\%3E3.0.CO;2-G

Tabachnick, B., \& Fidell, L. (2007). Using multivariate statistics (5th ed.). New York: Pearson.

van de Mortel, T. (2008). Faking it: Social desirability response bias in self-report research. Australian Journal of Advanced Nursing, 25(4), 40-48.

Verardi, S., Dahourou, D., Ah-Kion, J., Bhowon, U., Tseung, C. N., Amoussou-Yeye, D., Adjahouisso, M., et al. (2010). Psychometric properties of the Marlowe-Crowne Social Desirability Scale in eight African countries and Switzerland. Journal of Cross-Cultural Psychology, 41(1), 19-34. doi: $10.1177 / 0022022109348918$

Welte, J. W., \& Russell, M. (1993). Influence of socially desirable responding in a study of stress and substance abuse. Alcoholism: Clinical and Experimental Research 17, 758-761. doi: 10.1111/j.15300277.1993.tb00836.x

West, S., Taylor, A., \& Wu, W. (2012). Model fit and model selection in structural equation modeling. In R. Hoyle (Ed.), Handbook of structural equation modeling (pp. 209-231). New York: The Guilford Press.

Historial do artigo

Recebido $\quad 10 / 07 / 2015$

Aceite $\quad 15 / 02 / 2016$

Publicado 06/2016 
Propriedades psicométricas da SDRS-5 